\title{
Translação do conhecimento na implementação do processo de enfermagem em
}

\section{unidade neonatal}

\author{
Translation of knowledge in the Implementation of the nursing process in a neonatal unit \\ Traducción de conocimientos en la implementación del proceso de enfermería en una unidad
}

neonatal

Recebido: 16/06/2021 | Revisado: 21/06/2021 | Aceito: 26/06/2021 | Publicado: 11/07/2021

\author{
André Luiz Gomes de Oliveira \\ ORCID: https://orcid.org/0000-0002-5794-5684 \\ Hospital Federal dos Servidores do Estado, Brasil \\ E-mail: alaniteroi@gmail.com \\ Zenith Rosa Silvino \\ ORCID: https://orcid.org/0000-0002-2848-9747 \\ Universidade Federal Fluminense, Brasil \\ E-mail: zenithrosa52@gmail.com \\ Cláudio José de Souza \\ ORCID: https://orcid.org/0000-0001-7866-039X \\ Universidade Federal Fluminense, Brasil \\ E-mail: claudioenfo@gmail.com
}

\begin{abstract}
Resumo
O Objetivo deste estudo é socializar a vivência profissional da implementação do processo de enfermagem em uma unidade neonatal. Trata-se de um relato de experiência da implementação do processo de enfermagem em uma unidade neonatal de um hospital federal do Rio de Janeiro, ocorrido de maio de 2016 a dezembro de 2020, utilizando a técnica de translação do conhecimento. A implementação foi executada em três etapas: sensibilização da equipe e apresentação do formulário com três fases do processo de enfermagem (histórico, diagnósticos e intervenções de enfermagem); remodelação do formulário às cinco fases do processo de enfermagem e aprimoramento do formulário, permitindo a inclusão do campo de aprazamento e otimização do tempo de preenchimento. Essa sequência, possibilitou a implementação do processo de enfermagem na íntegra, agregando melhorias os registros de enfermagem e possibilitando qualificação dos cuidados prestados. A implementação do processo de enfermagem representa uma excelente ferramenta da gerência do cuidado, identificando as necessidades individuais dos RNs e consequentemente qualificando o cuidado.

Palavras-chave: Translação do conhecimento; Unidade de terapia intensiva neonatal; Cuidados de enfermagem; Enfermagem neonatal; Pesquisa em administração de enfermagem.
\end{abstract}

\begin{abstract}
The objective of this study is to socialize the professional experience of implementing the nursing process in a neonatal care unit. This is an experience report of the implementation of the nursing process in a neonatal care unit of a federal hospital in Rio de Janeiro, which took place from May 2016 to December 2020, using the knowledge translation technique. The implementation was carried out in three stages: sensitization of the team and presentation of the form with three phases of the nursing process (history, diagnoses and nursing interventions); remaking of the form for the five phases of the nursing process and improvement of the form, allowing the inclusion of medicine scheduling and optimization of filling time. This sequence enabled the full implementation of the nursing process, adding improvements to the nursing records and enabling the qualification of the care provided. The implementation of the nursing process represents an excellent care management tool, identifying the individual needs of newborns and consequently qualifying care.
\end{abstract}

Keywords: Translational medical research; Intensive care units, neonatal; Nursing care; Neonatal nursing; Nursing administration research.

\section{Resumen}

El objetivo de este estudio es socializar la experiencia profesional de implementar el proceso de enfermería en uma unidad neonatal. Se trata de un relato de experiencia de la implementación del proceso de enfermería en una unidad neonatal de un hospital federal de Río de Janeiro, que se llevó a cabo de mayo de 2016 a diciembre de 2020, utilizando la técnica de traducción del conocimiento. La implementación se llevó a cabo en tres pasos: conocimiento del equipo y presentación del formulario con tres fases del proceso de enfermería (historia, diagnósticos e 
intervenciones de enfermería); remodelación del formulario para las cinco fases del proceso de enfermería y mejora del formulario, permitiendo la inclusión del campo de programación y optimización del tiempo de llenado. Esta secuencia permitió la plena implementación del proceso de enfermería, agregando mejoras a los registros de enfermería y posibilitando la calificación de la atención proporcionada. La implementación del proceso de enfermería representa una excelente herramienta de gestión del cuidado, identificando las necesidades individuales de los recién nacidos y consecuentemente calificando el cuidado.

Palabras clave: Investigación en medicina traslacional; Unidades de cuidado intensivo neonatal; Atención de enfermería; Enfermería neonatal; Investigación en administración de enfermería.

\section{Introdução}

A lei $\mathrm{n}^{\circ} 7.486$ de 25 de junho de 1986, determina que ao enfermeiro cabe, privativamente, o planejamento da assistência de enfermagem (Brasil,1986). Esta determinação, remete-nos enquanto profissionais de enfermagem às responsabilidades gerenciais do enfermeiro, o que corrobora com o pensamento de que, para que o cuidado de enfermagem seja prestado adequadamente, deve-se preceder, antes de qualquer coisa, seu planejamento.

Em 2001, o então ministro da saúde José Serra, mediante a criação da Política Nacional de Humanização da Atenção Hospitalar (PNHAH), afirmou que: "com condições idênticas de trabalho, um hospital consegue melhores resultados que o outro, se houver compromisso da liderança, qualidade na gestão, competência e criatividade da equipe" (Brasil, 2001). Clarifica-se portanto, a importância do gerente à qualificação do cuidado de enfermagem, capaz de desencadear nos profissionais de enfermagem, reflexão e revisão de sua prática, rumo à adesão e comprometimento com a produção de cuidados à saúde (Silva, 2009). Complementando o pensamento, citamos um estudo que teve como objetivo avaliar a qualidade dos registros de enfermagem em papel versus registro eletrônico. O estudo concluiu que "ambas as formas de documentação revelaram desvantagens em termos de conteúdo, processo e estrutura" e sugere ainda, que tais resultados podem orientar gestores na identificação de estratégias visando melhorar a qualidade da documentação de enfermagem (Akhu-Zaheya, Al-Maaitah, \& Bany Hani, 2018).

Estas proposições evidenciam as atribuições do gerente de enfermagem, enquanto agente de mudança no processo de saúde-doença de sua unidade, com reflexo imediato sobre a saúde do cliente, família, comunidade, visibilidade e reconhecimento da equipe de enfermagem. Neste sentido, o Conselho Federal de Enfermagem (COFEN), aperfeiçoou as diretrizes operacionais à implementação e execução do processo de enfermagem (PE), por meio da Resolução COFEN ${ }^{\circ}$ 358/2009 (Brasil, 2009), a fim de proporcionar amparo legal e uma ferramenta de linguagem uniformizada do planejamento e qualificação dos cuidados de enfermagem, em quaisquer que sejam as áreas de atuação da equipe de enfermagem sejam elas públicas ou privadas. A presente resolução define as cinco etapas de execução do PE: histórico de enfermagem; diagnóstico de enfermagem; planejamento de enfermagem; implementação e avaliação de Enfermagem (Brasil, 2009). Todas requerem registro acurado, uma vez que representam atividades desenvolvidas no cuidado do paciente, implicando aspectos profissionais e legais para o enfermeiro, equipe multiprofissional e paciente (Azevedo at al., 2019).

Historicamente o PE surgiu nos Estados Unidos, na década de 1950, inicialmente como guia para os estudantes de enfermagem e posteriormente nos serviços de enfermagem para dar maior autonomia à profissão, favorecer o cuidado holístico e proporcionar a continuidade da assistência (Oliveira \& Peres, 2021). No Brasil, teve seu início na década de 1970 através dos estudos precursores de Wanda de Aguiar Horta que se embasou na teoria da motivação humana de Maslow, autor que postulou que todo ser humano possui necessidades comuns e hierarquizadas. Essas necessidades servem de sustentação do postulado teórico de Horta, arcabouço teórico à execução do PE (Lopes, 2000). O PE favoreceu a emancipação da profissão e qualificação profissional. Bem como, quando utilizado baseado em uma teoria, garante maior segurança na assistência de enfermagem e respaldo científico (Prado at al., 2019).

São inúmeras as dificuldades na aplicação do PE, passando pela deficiência de pessoal, falta de recursos materiais, 
além da escassez de capacitação da equipe para executá-lo. Autores conceituados afirmam ainda que há dificuldade para estabelecer o diagnóstico de enfermagem, atribuindo como causas o desconhecimento dos sinais e sintomas, das necessidades básicas alteradas e da nomenclatura destas necessidades, entre outras (Lopes, 2000; Azevedo at al., 2019). Um estudo realizado em unidades de saúde administradas pelo Estado de São Paulo, demonstrou que de 416 setores estudados, apenas 288 registravam quatro das cinco etapas do PE, fato que ratifica o pensamento de que mesmo sendo reconhecido como uma ferramenta importante para a gerência do cuidado do paciente e para a própria afirmação da profissão enquanto ciência, há necessidade de se implementar diversas estratégias para que se amplie a implementação do PE no Brasil (Oliveira \& Peres, 2021).

Face às diretrizes da legislação, cabe às instituições de saúde implementar e executar o PE. Uma alternativa para que as instituições de saúde atendam à legislação e efetive a implementação do PE é a translação do conhecimento (TC).

A TC é definida como: "um processo dinâmico, interativo, que inclui a síntese, divulgação, intercâmbio e a ética no conhecimento para promoção da saúde, fornecimento de serviços e produtos de saúde com maior efetividade visando fortalecer o sistema de saúde" (Lupatini at al., 2019). O sucesso dessa técnica abarca um forte treinamento ou elemento de capacitação, que considere o contexto cultural, político e econômico e encoraje uma abordagem colaborativa entre pesquisadores e tomadores de decisão (Andrade \& Pereira, 2019). Evidencia-se na TC, uma excelente oportunidade e ferramenta gerencial de qualificação do cuidado de saúde, em especial no cuidado de enfermagem, uma vez, que a partir desta ferramenta, as instituições de saúde poderão se valer de produtos científicos de inovação oriundos da academia e por conseguinte, remodelar as práticas de enfermagem (Lopes, 2000; Lupatini at al., 2019).

Diante ao exposto, este estudo tem como objetivo: socializar a vivência profissional quanto a implementação do Processo de Enfermagem em uma unidade neonatal.

\section{Metodologia}

Trata-se de um estudo do tipo relato de experiência a partir da vivência profissional dos autores. O projeto de implementação foi iniciado em maio de 2016 com etapas subsequentes e teve sua última versão em dezembro de 2020. O objetivo foi implementar o PE em uma unidade neonatal, utilizando-se como técnica a Translação do Conhecimento (TC). Caracterizando o cenário da experiência

O cenário de intervenção foi a unidade neonatal de um hospital público federal de grande porte, localizado na Cidade do Rio de Janeiro, composta pela Unidade de Terapia Intensiva (UTI) e pela Unidade de Cuidados Intermediários neonatal (UI). São oito leitos de UTI e doze de UI, 100\% do Sistema Único de Saúde (SUS) e regulados pelo sistema de regulação de leitos do Estado do Rio de Janeiro. A unidade recebe crianças oriundas de diversas cidades do Estado e com patologias diversificadas que vão da prematuridade, patologias congênitas cirúrgicas, cardíacas e de sistema gastrintestinal, além, dos recém-nascidos (RNs) oriundos da própria maternidade do hospital. Colaboram à internação do RN, condições socioambientais maternas, como a ocorrência de diabetes, obesidade mórbida, portadoras do vírus da Imunodeficiência humana, cardiopatias, entre outras.

Compõe a equipe: médicos, fisioterapeutas, nutricionistas, uma fonoaudióloga, uma terapeuta ocupacional e, por ser uma unidade de ensino e pesquisa, conta com residentes médicos e enfermeiros. Quanto à enfermagem, em média, três enfermeiros e três técnicos de enfermagem plantonistas por turno de doze horas em escala de revezamento.

Relatando a implementação da experiencia na unidade neonatal

O projeto foi elaborado para ser implementado em etapas e ampliando seu conteúdo ao longo da execução e aprendizado por parte da equipe de enfermeiros e técnicos de enfermagem. Utilizou-se como base à implementação o produto 
intitulado Manual de orientação de Diagnósticos e Intervenções de Enfermagem, sendo este, produto da dissertação cujo título foi Diagnósticos e Intervenções de Enfermagem em Pediatria: manual de orientação, defendido pelo primeiro autor e orientado pela segunda autora.

\section{$1^{\text {a }}$ Etapa: Sensibilização da equipe e apresentação do primeiro formulário de três fases}

Foram convidados para participar de rodas de conversa, todos os enfermeiros e técnicos de enfermagem, por meio de cartazes afixados no quadro de gestão à vista. A roda de conversa é um método onde os participantes podem se expressar, ouvir e ser ouvido, promovendo uma construção coletiva, pelo compartilhamento de experiências (Crossetti \& Góes, 2017). As rodas de conversa ocorreram em seis momentos distintos, pois, são três equipes no turno do dia e três à noite. Neste momento, a proposta foi ouvir os profissionais sobre o conhecimento e a experiência que possuíam a respeito do PE. Percebeu-se que todos os enfermeiros possuíam conhecimento sobre o PE, no entanto, não haviam praticado formalmente na unidade neonatal. Entre os técnicos, nem todos o conheciam, embora, alguns já utilizasse em outras instituições. Não houve tratamento estatístico quanto às características de gênero, nem formação, uma vez, que não representa objeto de estudo.

Após ouvir sobre seu conhecimento, foram apresentadas as estratégias de implementação do PE. Optou-se por iniciar a experimentação de vivenciar a execução de apenas três etapas do PE. Para tal, previamente criou-se um formulário digital e salvos em arquivo do Microsoft Word ${ }^{\circledR}$, nomeado com a identificação do RN, no diretório C do computador de uso da enfermagem. Contemplou as etapas de histórico, diagnósticos de enfermagem e intervenções de enfermagem, além dos dados de identificação do paciente, número de prontuário, diagnóstico clínico e idade cronológica. Tratava-se de um formulário em uma única folha.

A execução por parte da equipe obedeceu a seguinte dinâmica: o enfermeiro realizava o exame físico, identificando os problemas de saúde que requeriam cuidados de enfermagem e posteriormente, ia ao computador, acessava o arquivo do formulário do PE do paciente em atendimento e, realizava o registro, convertendo o exame à linguagem do histórico de enfermagem. Diante destes achados, definia os diagnósticos e as intervenções de enfermagem que a equipe deveria executar ao longo das vinte quatro horas de assistência.

Após o preenchimento inicial, o arquivo era salvo no computador, impresso e adicionado ao prontuário impresso do paciente. No dia seguinte, o enfermeiro responsável pelo paciente realizava a mesma dinâmica, alterando o arquivo salvo no dia anterior, de acordo com o novo exame realizado, salvava novamente no computador e imprimia o formulário alterado para execução pela equipe. Dessa forma, criou-se um arquivo dia, para o registro, não sendo, portanto, um arquivo cronológico sequencial, como se realiza em um prontuário eletrônico, uma vez, que os dados anteriores, são sempre excluídos com o novo exame do paciente (Figura 1). 
Figura1: Formulário inicial com 3 etapas do PE.

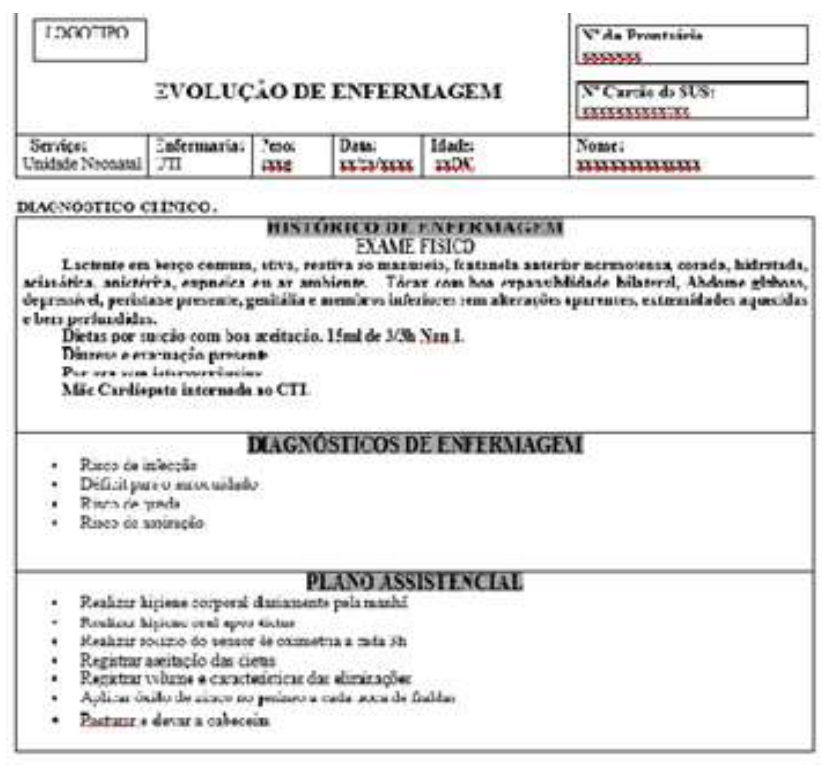

Fonte: os autores.

\section{Etapa: Remodelando o formulário às cinco etapas do PE}

Realizada nova roda de conversa com a equipe de enfermagem da unidade neonatal, a fim de que o novo formulário contendo as cinco etapas do PE fosse apresentado e avaliado. Em dezembro de 2016, passou-se a utilizá-lo para executar o PE e, dessa forma, facilitar a documentação dos registros e qualificação dos cuidados de enfermagem. Ainda se trata de um formulário em folha única e seria necessário um período de experimentação e avaliação prática dos profissionais para que se pudesse avançar para a etapa de aperfeiçoamento do formulário. O processamento do registro e dinâmica de realização foi alterado, pois, nesta fase, o enfermeiro precisou pensar em realizar as cinco etapas do PE. No formulário, foram inseridas as etapas de planejamento e avaliação de enfermagem. Por se tratar de uma nova experimentação pela equipe, a sequência das etapas foi direcionada por setas para a etapa seguinte a ser preenchida. Essa fase perdurou até janeiro de 2018, quando o formulário foi aprimorado (Figura 2).

Figura 2: Segundo formulário com 5 etapas do PE.

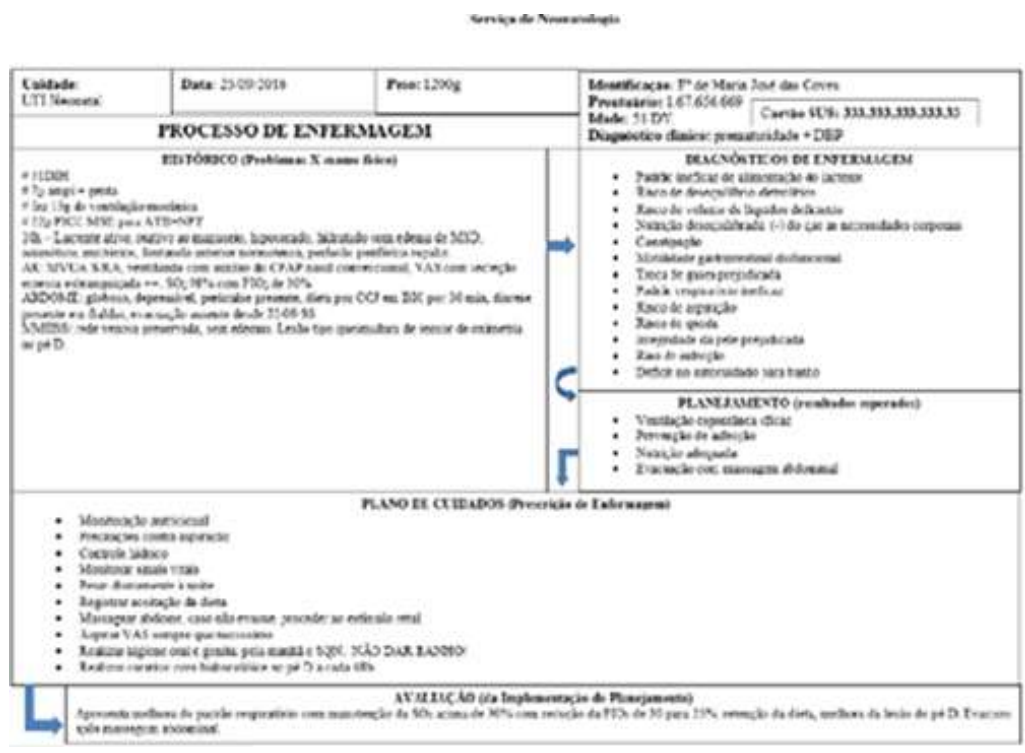

Fonte: os autores. 
Research, Society and Development, v. 10, n. 8, e23110817263, 2021

(CC BY 4.0) | ISSN 2525-3409 | DOI: http://dx.doi.org/10.33448/rsd-v10i8.17263

\section{Etapa: Aprimorando o formulário com as cinco etapas do PE}

Nesta etapa, iniciada em janeiro de 2018, a equipe já estava bem familiarizada com a rotina de realizar o PE, uma vez que, mantiveram-se estáveis em sua composição. A técnica utilizada para orientar os profissionais foi a divulgação de um tutorial, por WhatsApp® ${ }^{\circledR}$, através do grupo dos profissionais de enfermagem da unidade neonatal. Neste tutorial, foi divulgado a versão de duas folhas do formulário, que serão descritas abaixo (Figuras 3 e 4).

Figura 3: Formulário da fase 3 lado A.

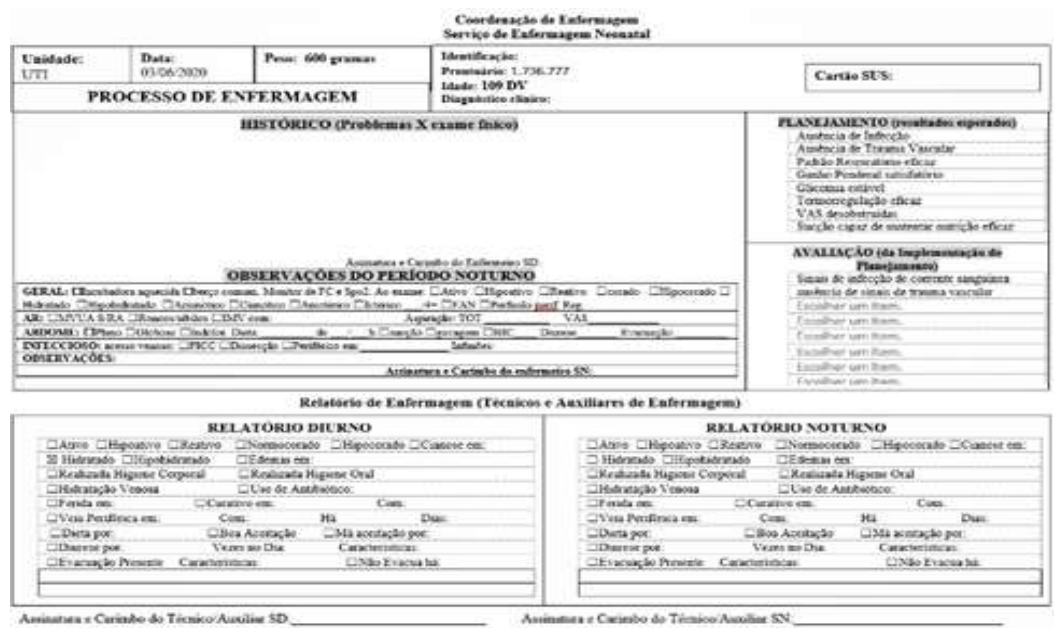

Fonte: os autores

Figura 4: formulário da fase 3 lado B.

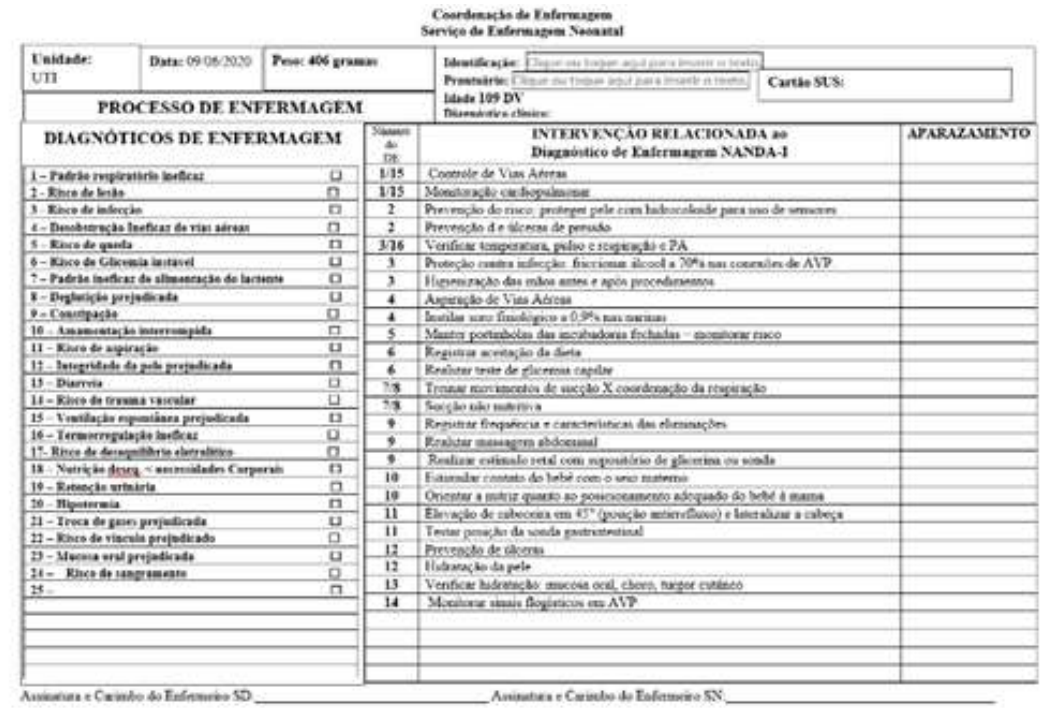

Fonte: os autores.

Seleção de unidade UTI/UI: inseriu-se uma lista suspensa, binária, onde o enfermeiro seleciona qual das unidades de internação o paciente se encontra internado, clicando em UTI ou em UI.

Identificação: inseridas caixas de texto simples, para que fosse preenchido livremente nome, número do prontuário, peso e diagnóstico clínico; no campo idade cronológica, criou-se uma lista suspensa com a contagem da idade em dias de vida, do um ao cento e oitenta, pois é a média máxima de tempo em que um RN fica internado em nossa unidade neonatal. Neste caso, o profissional clica na lista e seleciona a idade atualizada do paciente. 
Diagnósticos de Enfermagem (DE): foram mantidos os mesmos DE da versão anterior do formulário, obtidos do Manual de Diagnósticos e Intervenções de Enfermagem (Oliveira, 2015; Oliveira \& Silvino, 2017). Cada DE possui um número de identificação, de acordo com a sua inserção no formulário, bem como, uma caixa de seleção. Ao clicar na caixa, o DE é selecionado. Permanecem linhas adicionais para a livre inclusão de outros DE que o examinador identificar no RN, em sua avaliação. Para facilitar o agrupamento das intervenções de enfermagem, criou-se uma coluna com a interseção entre diversos DEs que requeiram as mesmas intervenções, como por exemplo, a verificação dos sinais vitais, pode ser definida como intervenção tanto para padrão respiratório ineficaz quanto para risco de infecção, dessa forma, nesta coluna de interseção, são incluídos os números de identificação de ambos os DEs.

Planejamento e avaliação: Optou-se por inserir caixas de texto suspenso com planejamento e avaliação para alguns dos DE da relação, deixando-se igualmente à coluna dos DEs, linhas para livre inserção de planejamento e avaliação de outros DE que tenham sido identificados no histórico do paciente. A exemplo, para o DE risco de trauma vascular, na caixa de planejamento, foi inserido ausência de trauma vascular e na caixa de avaliação, opções: ausência sinais de trauma vascular, sinais de trauma vascular.

Intervenções de Enfermagem: estão listadas algumas intervenções de enfermagem correspondente aos DEs elencados na coluna de DEs. Também possui linhas adicionais para livre inclusão de novas intervenções que se fizerem necessárias, de acordo com a avaliação do enfermeiro prescritor. Nesta versão do formulário, foi inserida a coluna de aprazamento dos cuidados e registro de observações pertinentes.

Registro dos técnicos e avaliação do enfermeiro do plantão noturno: nestes campos, trabalhou-se com caixas de Check-list onde os profissionais de forma manuscrita, assinalam seus registros, uma vez, que depois que o enfermeiro do plantão diurno preenche o formulário, este é impresso e anexado ao plano terapêutico do paciente. Todas as reavaliações e alterações a partir deste momento, portanto, seguem essa logística de registro manuscrito.

\section{Resultados e Discussão}

O prontuário do paciente é um documento que corresponde à memória escrita da pessoa sob cuidados, o qual consiste em registros identificatórios, sociodemográficos, clínicos, entre outros. Este é indispensável na comunicação entre a equipe de saúde e o paciente, bem como para a segurança, continuidade, eficácia e qualidade da assistência (Peres, Jensen \& Martins, 2016). Na enfermagem, o cotidiano do processo de trabalho gera, a todo o momento, dados decorrentes das atividades assistenciais, gerenciais, de ensino e de pesquisa (Medeiros \& Ribeiro, 2015). Evidenciamos dessa forma, que o prontuário do paciente é a documentação principal de todos os cuidados prestados pela equipe multidisciplinar, nele são registradas decisões, condutas e as respostas do paciente ao atendimento da equipe, sendo a equipe de enfermagem a principal produtora destes registros.

Entre as diversas maneiras de otimizar o cuidado de enfermagem, pode-se apontar a utilização da tecnologia da informação e a implementação da SAE” (Medeiros \& Ribeiro, 2015). A documentação representa a única maneira viável e segura de conhecer, de forma sistemática, o que foi realizado e, que pode ser facilitado com o uso de programas computacionais (Azevedo at al., 2019). Trata de um documento chave ao acompanhamento da saúde do paciente, bem como, base à formação dos profissionais e aprimoramento das ciências da saúde. Quanto maior a sistematização e clareza nas informações, melhores serão as condutas ao paciente, da mesma forma, quando um registro é informatizado, aumenta a segurança no cuidado do paciente, minimizando a possibilidade de ocorrência de eventos adversos, principalmente, quanto à prescrição e manipulação e administração de medicamentos.

Foi possível identificar uma acentuada melhora nos registros de enfermagem na unidade neonatal. Tal melhora 
traduziu-se pela maior assertividade e clareza no registro, uma vez, que antes da implementação do PE, estes eram realizados em formulário, simples de evolução manuscrita. Evidências científicas mostraram, na ótica dos profissionais de enfermagem, que a implantação da SAE possibilitou uma melhora na qualidade da assistência prestada aos clientes (Pissaia at al., 2017). Segundo Cruz, a documentação de enfermagem nos serviços de saúde, quando estruturada de acordo com o PE, permite estimar como o processo é operacionalizado pelos enfermeiros e, pelo menos em parte, a qualidade do cuidado de enfermagem oferecido ao paciente (Cruz at al., 2016).

Há uma preocupação mundial em relação à prevenção de erros na assistência à saúde. Pesquisas demonstram um gasto de bilhões de dólares, ocasionado por erros evitáveis no cuidado ao paciente. A partir de 1999 iniciaram-se as ações de combate aos erros evitáveis na assistência à saúde, culminando com a iniciativa de se implantar protocolos direcionados à segurança do paciente. O marco divisório comportamental foi a divulgação do relatório do instituto de medicina dos Estados Unidos, intitulado "errar é Humano" (Kohn at al., 2000), ao qual apontava a lacuna de cuidado com a segurança do paciente. Esses protocolos ressoaram pelo mundo. No Brasil, em 2013, o ministério da saúde instituiu o Programa Nacional de Segurança do Paciente (PNSP) como uma ferramenta capaz de nortear e colaborar com o gerenciamento do cuidado e redução dos erros evitáveis na prestação de serviços de saúde (Silva, 219).

Um dos pilares do PNSP é a comunicação no ambiente dos serviços de saúde; neste pilar, portanto, se enquadram os registros dos profissionais, pois é o meio de comunicação entre os diversos disciplinas que cuidam do paciente (Brasil, 2013). Segundo Pissaia, tecnologias de informática aliadas à gestão do serviço integralizam a assistência ao cliente por meio da sistematização da assistência de enfermagem (SAE) e possibilitam a melhoria na comunicação entre a equipe (Pissaia at al., 2017). Vale ressaltar, que o PE está inserido na SAE, sendo, portanto, a ferramenta operacional para o cuidado individualizado.

A partir da implementação do PE, houve uma padronização da linguagem aplicada aos cuidados de enfermagem. A equipe passou a avaliar os pacientes entendendo a necessidade de se proceder a todas as etapas do PE e consequentemente contribuindo à qualificação do cuidado de enfermagem. Além disso, por tratar-se de uma unidade que recebe residentes de enfermagem, promove o treinamento destes ao raciocínio clínico e decisório sobre a terapêutica de enfermagem adequada ao paciente sob seus cuidados em consonância com o eixo referente à educação na Política Nacional de Atenção Hospitalar (Brasil, 2013).

\section{Conclusão}

A vivência nesta Unidade Neonatal possibilitou um olhar crítico e reflexivo acerca das contribuições do profissional enfermeiro para a efetivação do gerenciamento do cuidado, com vista à promoção de melhorias aos recém-nascidos. A partir da implementação do processo de enfermagem por meio da técnica de translação, foi possível favorecer a melhoraria da assistência de enfermagem. Como limitação do estudo, identificamos a abrangência deste, pois representa a realidade de uma unidade de internação, não possuindo maior amplitude.

Ainda como contribuição, sugere-se que outros serviços de saúde possam se apropriar deste relato de experiência, inspirando ações de planejamento, implementação e avaliação do processo de enfermagem, proporcionando reflexão das práticas de gerenciamento e qualificação do cuidado de enfermagem, bem como, alinhamento com a legislação de enfermagem e políticas de saúde.

\section{Referências}

Akhu-Zaheya, L., Al-Maaitah, R., \& Bany Hani, S. (2018). Qualidade da documentação de enfermagem: registros de saúde baseados em papel versus prontuários eletrônicos de saúde. Revista de enfermagem clínica, 27(3-4), e578-e589. https://doi.org/10.1111/jocn.14097. 
Andrade, K. R. C. de., \& Pereira, M. G. (2020). Knowledge translation in the reality of Brazilian public health. Revista de Saúde Pública, 54, 72. https://doi.org/10.11606/s1518-8787.2020054002073.

Azevedo, O. A., Guedes, E. S., Araújo, S. A. N., Maia, M. M., \& Cruz, D. A. L. M. (2019). Documentation of the nursing process in public health institutions. Rev Esc Enferm USP, 53, e03471. http://dx.doi.org/10.1590/S1980-220X2018003703471.

Crossetti, M. G. O., \& Góes M. G. O. (2017). Translação do conhecimento: um desafio para prática de enfermagem. Rev. Gaúcha Enferm, 38(2), e74266. http://doi.org/10.1590/1983-1447.2017.02.74266.

Cruz, D. A. L. M., Guedes, E. S., Santos, M. A., Sousa, R. M. C., Turrini, R. N. T., Maia, M. M., \& Araújo S. A. N. (2016). Nursing process documentation: rationale and methods of analytical study. Rev Bras Enferm, 69(1), 183-189. http://dx.doi.org/10.1590/0034-7167.2016690126i.

Kohn, L. T., Corrigan, J. M., \& Donaldson, M. S. (eds.). (2000). To err is human: building a safer health system. Washington, D.C.: National Academy Press.

Lei n. 7.498/86, de 25 de junho de 1986. (1986, 25 junho). Dispõe sobre a regulamentação do exercício da enfermagem e dá outras providências. COFEN Conselho Federal de Enfermagem. http://www.cofen.gov.br/lei-n-749886-de-25-de-junho-de-1986_4161.html.

Lima, L. M., \& dos Santos, S. R. (2015). Protótipo de um software para registro de enfermagem em unidade de terapia intensiva neonatal. Aquichan, 15(1), 31-43. https://doi.org/10.5294/aqui.2015.15.1.4.

Lopes, H. B. (2000, julho). Experiência de implantação do processo de enfermagem utilizando os diagnósticos de enfermagem (Taxionomia da NANDA), resultados esperados, intervenções e problemas colaborativos. Rev. Latino-Am. Enfermagem, 8(3), 115-118. https://doi.org/10.1590/S010411692000000300017

Lupatini, E. O., Barreto, J. O. M., Zimmermann, I. R., \& Silva, E. N. (2019, novembro). Medicamentos e pesquisa translacional: etapas, atores e políticas de saúde no contexto brasileiro. Saúde em Debate, 43(spe2), 181-199. https://doi.org/10.1590/0103-11042019S214.

Ministério da Saúde. (2001). Programa nacional de humanização da assistência hospitalar (Série C, Programas e relatórios n. 20). Secretaria de Assistência à saúde. Brasília, DF: Ministério da Saúde.

Oliveira, A. L. G. (2015). Diagnósticos e intervenções de enfermagem em pediatria. Dissertação de mestrado, Universidade Federal Fluminense, Niterói, RJ, Brasil.

Oliveira, A. L. G. D., \& Silvino, Z. R. (2017, agosto). Prevalência de diagnósticos de enfermagem NANDA-I em um hospital pediátrico. Nursing (São Paulo), 20(231), 1792-1796. https://pesquisa.bvsalud.org/portal/resource/pt/bde-31482.

Oliveira, N. B. D., \& Peres, H. H. C. (2021). Quality of the documentation of the Nursing process in clinical decision support systems. Revista LatinoAmericana de Enfermagem, 29, e3426. http://doi.org/10.1590/1518-8345.4510.3426.

Peres, H. H. C., Jensen, R., \& Martins, T. Y. D. C. (2016). Avaliação da acurácia diagnóstica em enfermagem: papel versus sistema de apoio à decisão. Acta Paulista de Enfermagem, 29(2), 218-224. https://doi.org/10.1590/1982-0194201600030.

Pissaia, L., Costa, A., Moreschi, C., Rempel, C., Carreno, I., \& Granada, D. (2018). O impacto das tecnologias na implantação da sistematização da assistência à enfermagem hospitalar: uma revisão integrativa. Revista de Epidemiologia e Controle de Infecção, 8(1), 92-100. https://doi.org/10.17058/reci.v1i1.8953

Portaria n. 3.390, de 30 de dezembro de 2013 (2013, 30 dezembro). Institui a Política Nacional de Atenção Hospitalar (PNHOSP) no âmbito do Sistema Único de Saúde (SUS), estabelecendo- se as diretrizes para a organização do componente hospitalar da Rede de Atenção à Saúde (RAS). Ministério da Saúde. https://bvsms.saude.gov.br/bvs/saudelegis/gm/2013/prt3390_30_12_2013.html.

Portaria n. 529, de $1^{\circ}$ de abril de 2013. (2013, 01 abril). Institui o programa nacional de segurança do paciente. Ministério da Saúde. https://bvsms.saude.gov.br/bvs/saudelegis/gm/2013/prt0529_01_04_2013.html.

Prado, N. C. D. C., Santos, R. S. D. C., Lima, D. M. D., Góis, M. M. D. C. D., Costa, R. H. S., \& Silva, R. A. R. D. (2019). Necessidades humanas básicas alteradas em neonatos com cateter central de inserção periférica. Rev. enferm. UERJ, 27, e44521-e44521. http://dx.doi.org/10.12957/reuerj.2019.44521.

Resolução n. 358 de 23 de outubro de 2009. (2009, 15 outubro). Dispõe sobre a sistematização da assistência de enfermagem e a implementação do processo de enfermagem em ambientes, públicos ou privados, em que ocorre o cuidado profissional de enfermagem, e dá outras providências. COFEN - Conselho Federal de Enfermagem. http://www.cofen.gov.br/resoluo-cofen-3582009_4384.html.

Silva, A. M. (2009). Competências para o gerenciamento da enfermagem: revisão da literatura. Monografia de graduação, Universidade Federal do Rio Grande do Sul, Porto Alegre, RS, Brasil. 